\title{
AN EMBEDDING THEOREM FOR HOMEOMORPHISMS OF THE CLOSED DISC
}

\author{
GARY D. JONES
}

\begin{abstract}
If $f$ is an orientation preserving self-homeomorphism of the closed disc $D$ with the property that if $x, y \in D-N$, where the set of fixed points $N$ is finite and contained in $D$-int $D$, then there exists an arc $A \subset D-N$ joining $x$ and $y$ such that $f^{n}(A)$ tends to a fixed point as $n \rightarrow \pm \infty$, then it is shown that $f$ can be embedded in a continuous flow on $D$.
\end{abstract}

1. Preliminary remarks. Let $X$ be a topological space and let $G$ be a topological group. The ordered triple $(X, G, F)$ is a dynamical system if (1) $F: X \times G \rightarrow X$ is continuous, (2) $F\left(F\left(x, g_{1}\right), g_{2}\right)=$ $F\left(x, g_{1}+g_{2}\right)$, and (3) $F(x, e)=x$ for every $x \in X$. If $G$ is the additive group of real numbers, $(X, G, F)$ is called a continuous flow. If $G$ is the additive group of integers, then $(X, G, F)$ is called a discrete flow. A discrete flow determines and is determined by the homeomorphism $F(x, 1)$.

The problem of embedding a discrete flow in a continuous flow can be stated in the following way. Let $f$ be a self-homeomorphism of a topological space $X$. Find a continuous flow $(Y, R, F)$ such that (1) $F(y, 1)$ is invariant on a subset $Z$ of $Y$, (2) $Z$ is homeomorphic to $X$, and (3) $F(y, 1)$ on $Z$ is topologically equivalent to $f$ on $X$, i.e. there is a homeomorphism $h: X \rightarrow Z$ such that $h^{-1}(F(h(x), 1))=f(x)$.

If the space $X$ is allowed to be enlarged in order to accommodate $F$, the problem is referred to as the unrestricted problem. If $X=Y=Z$, the problem is referred to as the restricted problem.

The unrestricted problem is easily solved [5], [7], [8] for any topological space $X$ and any self-homeomorphism. The two solutions cited in [5] are shown in [9] to be topologically equivalent if and only if the homeomorphism has no fixed points.

The restricted problem is only partially solved. In [2] and [6] it is proved that a self-homeomorphism $f$ of an interval can be embedded in a continuous flow if and only if it is order preserving. In [5] necessary and sufficient conditions for a self-homeomorphism of a simple closed curve to be embedded in a continuous flow are given. In [3] and [9] sufficient conditions for a self-homeomorphism of the closed disc to be embedded in a continuous flow are given. In [1],

Received by the editors February 16, 1970.

AMS 1969 subject classifications. Primary 5482; Secondary 2240, 5480.

Key words and phrases. Embedding, discrete flows, continuous flows, homeomorphisms, closed disc. 
[4], [9] sufficient conditions for a self-homeomorphism of the plane to be embedded in a continuous flow are given.

It is easily shown that a necessary condition for a self-homeomorphism of a subset of the plane to be embedded in a continuous flow is that it be orientation preserving. Thus, throughout this paper $f$ will denote such a homeomorphism.

2. Embedding theorem. Let $f$ be an orientation preserving selfhomeomorphism of the closed disc $D$. It is well known that $f$ must have at least one fixed point. If in addition $f$ has the property that if $x, y \in D-N$, where the set of fixed points $N$ is finite and contained in $D$-int $D$, then there exists an arc $A \subset D-N$ joining $x$ and $y$ such that $f^{n}(A)$ tends to a fixed point as $n \rightarrow \pm \infty$, then it is easy to show that there exist at most two fixed points.

THEOREM. If $f$ is a homeomorphism as described above, then $f$ can be embedded in a continuous flow. [9].

Proof. The case where $f$ has exactly one fixed point is proved in

Suppose $f$ has two fixed points $x_{1}$ and $x_{2}$. Suppose without loss of generality $D=\left\{(x, y): x^{2}+y^{2} \leqq 1\right\}$ in the plane $P_{1}$. Suppose $x_{1}=(0,1)$ and $x_{2}=(0,-1)$. Consider the set $S=P_{1}-\{(x, y): x=0,|y| \geqq 1\}$. This set $S$ is homeomorphic to a plane $P_{2}$. Suppose $g_{1}: S \rightarrow P_{2}$ is a homeomorphism where $\left\{(x, y): x^{2}+y^{2}=1, x>0\right\}$ is mapped onto the $y$-axis in $P_{2}$. If $z$ is an element of the half-plane in $P_{2}$ defined by the $y$-axis not containing $g_{1}(D)$, then define a self-homeomorphism $T_{1}$ of the closed half-plane by

$$
T_{1}((x, y))=\left(x,\left(g_{1} f g_{1}^{-1}(0, y)\right)_{y}\right) .
$$

Suppose $g_{2}: S \rightarrow P_{3}$ is a homeomorphism where $\left\{(x, y): x^{2}+y^{2}=1, x<0\right\}$ is mapped onto the $y$-axis in plane $P_{3}$. If $z$ is an element of the halfplane in $P_{8}$ defined by the $y$-axis not containing $g_{2}(D)$ then define a self-homeomorphism $T_{2}$ of the closed half-plane by $T_{2}((x, y))=$ $\left(x,\left(g_{2} f g_{2}^{-1}(0, y)\right)_{y}\right)$. Now define $T: S \rightarrow S$ by

$$
\begin{aligned}
T(z) & =g_{1}^{-1} T_{1} g_{1}(z) & & \text { if } g_{1}(z) \in \text { domain of } T_{1}, \\
& =f(z) & & \text { for } z \in D, \\
& =g_{2}^{-1} T_{2} g_{2}(z) & & \text { if } g_{2}(z) \in \text { domain of } T_{2} .
\end{aligned}
$$

It is clear from the definition of $T$ that $T$ is an orientation preserving self-homeomorphism of $S$ with no fixed points. It is also clear that $g_{1} T g_{1}{ }^{-1}$ is a self-homeomorphism of $P_{2}$ with exactly one fundamental region, and is therefore topologically equivalent to a translation $\left[1\right.$, p. 71]. Thus, there is a curve $L \subset S$ such that $T^{j}(L)$ separates $S$ 
for every integer $j, T^{n}(L) \cap T^{m}(L)=\varnothing$ for $n \neq m$, if $z \in S, T^{k}(z)$ is in the strip bounded by $L$ and $T(L)$ for some unique integer $k$, and $g_{i}(L)$ intersects the $y$-axis in $P_{i+1}$ in exactly one point for $i=1,2$. Let $L_{1}=L \cap D$. The region bounded by $L_{1}, D$-Int $D$ from $L_{1}$ to $f\left(L_{1}\right), f\left(L_{1}\right)$, and $D$-Int $D$ from $f\left(L_{1}\right)$ to $L_{1}$ is homeomorphic to a rectangle with $f\left(L_{1}\right)$ and $L_{1}$ as opposite sides. Suppose $h$ is this homeomorphism. Now if $x \in L_{1}$ and if $0 \leqq t<1$ define $F_{1}(x, t)=y$ where $h(y)=(1-t) h(x)+t h(f(x))$. If $x \in L_{1}$ and $t$ is any real number, define $F_{2}(x, t)=f^{n}\left(F_{1}(x, s)\right)$, where $n+s=t, n$ is an integer, and $0 \leqq s<1$. If $x$ is in the interior of the strip bounded by $L_{1}$ and $f\left(L_{1}\right)$ or if $x \in L_{1}$, and if $t_{1}$ is any real number, define $F_{3}\left(x, t_{1}\right)=F_{2}\left(y, t_{2}+t_{1}\right)$ where $h(x)=\left(1-t_{2}\right) h(f(y))+t_{2}(h(y))$ and $y \in L_{1}$. If $x$ is any point in $D$ $-\left\{x_{1}, x_{2}\right\}$, define $F(x, t)=F_{3}\left(f^{-m}(x), t+m\right)$, where $f^{-m}(x)$ is in the strip bounded by $L_{1}$ and $f\left(L_{1}\right)$.

Now define $\sigma: D \times R \rightarrow D$ by

$$
\begin{aligned}
\sigma(x, r) & =F(x, r) & & \text { if } x \in D-\left\{x_{1}, x_{2}\right\} \\
& =x & & \text { otherwise. }
\end{aligned}
$$

It is not difficult to show that $\sigma$ is a continuous flow, and that $\sigma(x, n)=f^{n}(x)$ for every $x \in D$ and every in teger $n$.

\section{REFERENCES}

1. S. A. Andrea, On homeomorphisms of the plane which have no fixed points, Abh. Math. Sem. Univ. Hamburg 30 (1967), 61-74. MR 34 \#8397.

2. N. J. Fine and G. E. Schweigert, On the group of homeomorphisms of an arc, Ann. of Math. (2) 62 (1955), 237-253. MR 17, 288.

3. N. E. Foland, An embedding theorem for discrete flows on a closed 2-cell, Duke Math. J. 33 (1966), 441-444. MR $33 \# 6609$.

4. - An embedding theorem for contracting homeomorphisms, Math. Systems Theory 3 (1969), 166-169.

5. N. E. Foland and W. R. Utz, The embedding of discrete flows in continuous flows,

Proc. Internat. Sympos. Ergodic Theory (Tulane Univ., New Orleans, La., 1961), Academic Press, New York, 1963, pp. 121-134. MR 28 \#3412.

6. M. K. Fort, Jr., The embedding of homeomorphisms in flows, Proc. Amer. Math. Soc. 6 (1955), 960-967. MR 18, 326.

7. W. H. Gottschalk, Minimal sets: an introduction to topological dynamics, Bull. Amer. Math. Soc. 64 (1958), 336-351. MR $20 \# 6484$.

8. W. H. Gottschalk and G. A. Hedlund, Topological dynamics, Amer. Math. Soc. Colloq. Publ., vol. 36, Amer. Math. Soc., Providence, R. I., 1955. MR 17, 650.

9. G. D. Jones, The embedding of flows in flows, Ph.D. Thesis, Univ. of Missouri, Columbia, 1969.

10. W. R. Utz, The embedding of a linear discrete flow in a continuous flow, Colloq. Math. 15 (1966), 263-270. MR 34 \#2000.

Murray State University, Murray, Kentucky 42071 